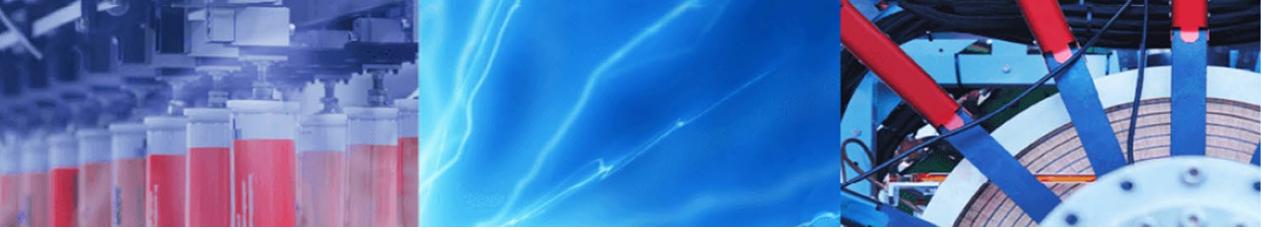

Research Article

\title{
Development and characterization of nanosheets attached nanotetrapods of zinc oxide
}

\author{
Jasbir Singh ${ }^{1} \cdot$ Sukhbir Singh ${ }^{2} \cdot$ Sitender Singh ${ }^{3} \cdot$ Devender Singh $^{3}$ (ID
}

(c) Springer Nature Switzerland AG 2019

\begin{abstract}
A unique morphology of $\mathrm{ZnO}$ nanostructures namely nanosheets attached nanotetrapods have been grown using thermal evaporation of zinc, which promises innovative advantages of both one and two dimensional nanostructures. The nanostructures have been characterized using X-ray Diffractometer, Scanning Electron Microscope, Transmission Electron Microscope, Energy Dispersive X-ray Analysis and Photoluminescence (PL) techniques. Various effects of low dimensionality have been analysed. Indexing of various peaks in XRD analysis confirmed the typical wurtzite $\mathrm{ZnO}$ structure. The SEM analysis revealed an interconnected three dimensional network of nanotetrapods with two dimensional sheets attached to the joints of nanotetrapods and a possible size control mechanism. TEM analysis provided a better understanding of the general morphology of nanotetrapods and size of nanorods and nanosheets. The chemical composition of the grown product has been analysed by EDAX which rules out the presence of any impurity and confirmed the purity of $\mathrm{ZnO}$ nanostructures. The PL spectroscopy was used to study the optical properties which indicate high optical purity of materials. The crystallite size as estimated by XRD analysis is observed in good agreement with the PL examination.
\end{abstract}

Keywords Zinc oxide $\cdot$ Nanotetrapods $\cdot$ Nanosheets $\cdot$ Thermal evaporation $\cdot$ Morphology

\section{Introduction}

Zinc oxide is a multifunctional technological material owing to its very peculiar physical, chemical and biocompatible properties. ZnO falls under the category of II-VI compound of semiconductors and is a borderline case between the covalent and ionic semiconductors. $\mathrm{ZnO}$ crystals generally stabilize in the wurtzite structure, whereas it exhibits the rock salt phase at high pressure. $\mathrm{ZnO}$ in wurtzite structure lacks a centre of symmetry which gives rise to its piezoelectric and pyroelectric properties. $\mathrm{ZnO}$ shows $3.37 \mathrm{eV}$ direct band gap and $60 \mathrm{meV}$ exciton binding energy. Such exceptionally large binding energy may lead to room temperature excitonic emission and consequently $\mathrm{ZnO}$ could be a high potential photonic material. Also the combination of low resistivity and high optical transmittance in visible region of spectrum makes it suitable for transparent and conducting contacts. $\mathrm{ZnO}$ shows another fascinating property of piezoelectricity due to the non-centrosymmetric crystal structure. External pressure induced lattice distortions in $\mathrm{ZnO}$ leads to the displacement of the centre of negative and positive charge. This results into the appearance of a net dipole moment over the entire crystal which in turn facilitates a very high piezoelectric tensorand. $\mathrm{ZnO}$ exhibits excellent piezoelectric and pyroelectric properties as a direct consequence. Novel applications such as piezoelectric nanogenerators based on zinc oxide nanowire arrays have been reported [1]. Ferromagnetism has also been reported in $\mathrm{ZnO}$ when doped with $\mathrm{Mn}, \mathrm{Fe}$ and $\mathrm{Co}$. $\mathrm{ZnO}$ is also a key material in gas sensing and shows highest sensitivity among various

\footnotetext{
$\triangle$ Devender Singh, devjakhar@gmail.com | ${ }^{1}$ Department of Physics, Government College, Dujana, Jhajjar 124102 , India. ${ }^{2}$ Department of Physics, University Institute of Engineering and Technology, Maharshi Dayanand University, Rohtak 124001, India. ${ }^{3}$ Department of Chemistry, Maharshi Dayanand University, Rohtak, Haryana 124001, India.
}

SN Applied Sciences (2019) 1:912 | https://doi.org/10.1007/s42452-019-0957-7

Received: 2 April 2019 / Accepted: 20 July 2019 / Published online: 24 July 2019 
metal oxides barring $\mathrm{SnO}_{2}$. When material is exposed to a gaseous species, it reacts with the adsorbed surface oxygen and alters its conductivity. The change in conductivity is used as a measure of gas concentration [2]. In the last couple of decades nanotechnology has seen explosive growth and zinc oxide is one of the front runners in this area too [3]. The reduction in the size result in novel and tailorable mechanical, chemical and optoelectric properties, which are mainly attributed to enhanced surface to volume ratio and quantum confinement. $\mathrm{ZnO}$ is a versatile material in terms of nanostructured growth morphologies and is one of the richest known family of nanostructures such as nanoparticles, nanowires, nanobelts, nanotubes, nanorings, nanotetrapods, nanonails, nanoclips, nanosheets and a variety of other nanostructures [4-8].

Owing to these properties, $\mathrm{ZnO}$ has become a very promising material for many practical applications such as battery anodes [9] dye sensitized [10] and perovskite solar cells [11], thin films gas sensors [2], implants due to biocompatibility [12], antibacterial and biomedical applications [13-15], smart clean surfaces using superhydrophobicity [16], biosensors [17], solar photocatalysts [18], flame retardants [19] and water splitting [20]. Zinc oxide nanotetrapod is a very fascinating morphology popularly known as $\mathrm{T}-\mathrm{ZnO}$ in literature, which is advantageous in several ways [21]. This peculiar morphology basically have four interconnected nanowires, hence present itself for all the conventional uses of nanowires, but due to its structure it always forms a three dimensional nanostructure presenting a large surface to volume ratio which is considered so crucial for applications like chemical sensors. Numerous synthesis methods have been employed to grow zinc oxide nanotetrapods including hydrothermal, microwave, combustion method, thermal evaporation, CVD and T-ZnO have been successfully tested in varied applications such as solar cells [22], biomedical [23], gas sensing [24], UV sensors [25], field emission [26], hydrophobicity and as composite fillers [27]. $\mathrm{ZnO}$ based materials also have shown very promising properties superior to commonly observed morphologies such as nanorods, nanoparticles and nanocrystalline thin films.

In the current study, distinct morphological nanosheets have been grown by a simple, inexpensive and novel method with an element of size control of the nanostructures synthesized. Three different sized nanosheets attached T-ZnO was grown by using the modified thermal evaporation process. The grown structures have been investigated with various characterization techniques. From these investigations the high crystallinity of nanostructures and the wurtzite phase of $\mathrm{ZnO}$ have been identified. The photoluminescence study has been applied to analyse the optical properties. The variation of different properties with size of nanostructures has been discussed.

\section{Experimental}

\subsection{Synthesis of T-ZnO}

The experimental procedure used for the growth of $\mathrm{T}-\mathrm{ZnO}$ is shown in the schematic Fig. 1. In present process $99.99 \%$ pure pieces of zinc metal (Sigma Aldrich) were placed in a tungsten wired basket inside bell jar of a vacuum coating unit. The basket was first vacuum baked to ensure the removal of residual impurities. A p-type cleaned Si substrate was placed $1.5 \mathrm{~cm}$ above the basket for the fabrication of sample. The basket was heated for 1 min resulting in the burning of the zinc metal with flames. Temperature of the flame was $950^{\circ} \mathrm{C}$. The chamber was opened after $15 \mathrm{~min}$ and various products were obtained. A cottony product was collected from the floor of the coating unit, it was also observed during the burning process that a web type material was dispersed in air inside the bell jar

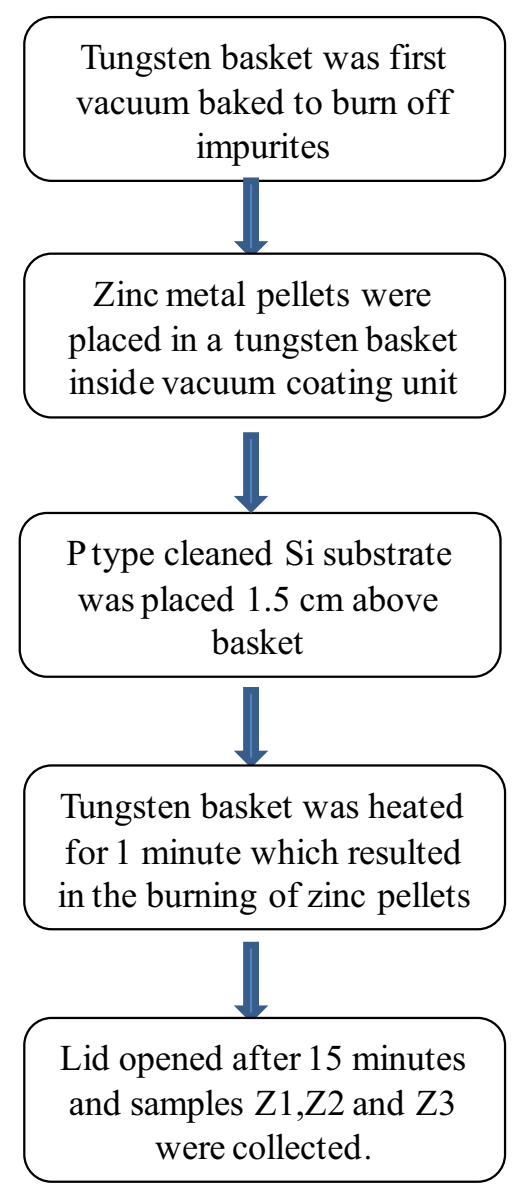

Fig. 1 Schematics of the experiment 
and later settled. The material was named as Sample Z1. The powder found swelled upon the tungsten basket was categorized as Sample Z2. The silicon substrate got deposited with a white paste and labelled as Z3. The macroscopic morphologies of samples were appeared dissimilar for different samples. Also, the position of collection of three types of grown material hinted about their density. As it later became clear after the detailed analysis that as the distance from the basket increased the size of $\mathrm{T}-\mathrm{ZnO}$ decreased accordingly with the web like powder being the smallest T-ZnO. As the products could be obtained on arbitrary substrates ( $Z 1$ was collected from the floor of the coating unit, Z2 was collected from the basket itself and Z3 although collected on Si substrate but the similar product was also found on the background lid), it could be easily theorised that substrate do not have any roll in the nucleation and growth.

\subsection{Instrumentation}

The synthesis of materials was carried out inside a vacuum coating unit HIND HIVAC No. 12D4D. X-ray diffraction was studied with CuKa line with X-ray diffractometer PW-1710 (Cu target, $40 \mathrm{kV}$ and $20 \mathrm{~mA}$ ). Microstructure of the products was inspected using JEOL JSM-5800 Scanning Electron Microscope (SEM). To achieve greater insight of morphology, the samples were examined by Transmission Electron Microscopy (TEM) using JEOL JEM 2010 Electron Microscope. The room temperature photoluminescence study was performed on Perkin Elmer LS 55 Luminescence spectrometer with $320 \mathrm{~nm}$ Xenon discharge lampas excitation source.

\section{Result and discussions}

\subsection{SEM study}

The morphology of three products $Z 1, Z 2, Z 3$ was analysed by scanning SEM. Although due to limitation of the resolution of SEM and smaller dimensions of the nanostructures of the samples, exact details of the morphology are not perceptible. Nevertheless the general overview of sample can be discerned.

Figure $2 a, b, c$ depicts the SEM images of sample $Z 1$ and $Z 3$ and $Z 2$ respectively. It is clear from the images that inspite of the higher magnification of image in Fig. 2a, the details of the sample are not clearly discernible. The micrographs are clear in both the sample Z3 (Fig. 2b) and sample Z2 (Fig. 2C). It is evident from the comparison of the images that although the dimension of the nanostructures is smallest in sample $\mathbf{Z 1}$, however the general morphology of all

Fig. 2 SEM images of sample Z1 (a), Z3 (b) and Z2 (c)

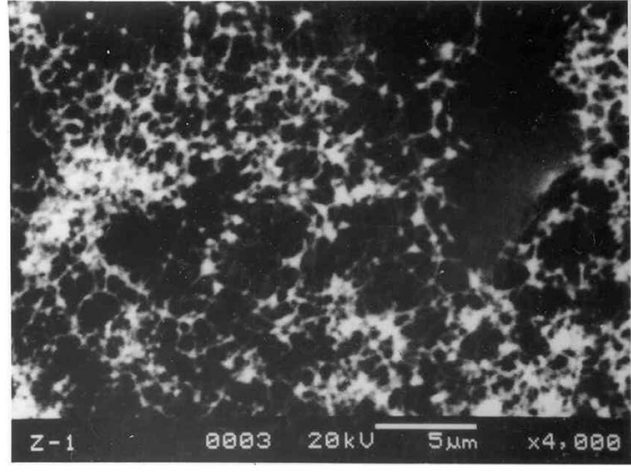

(a)

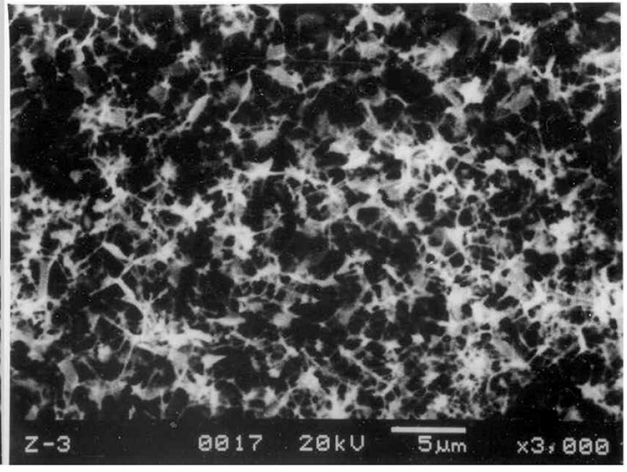

(b)

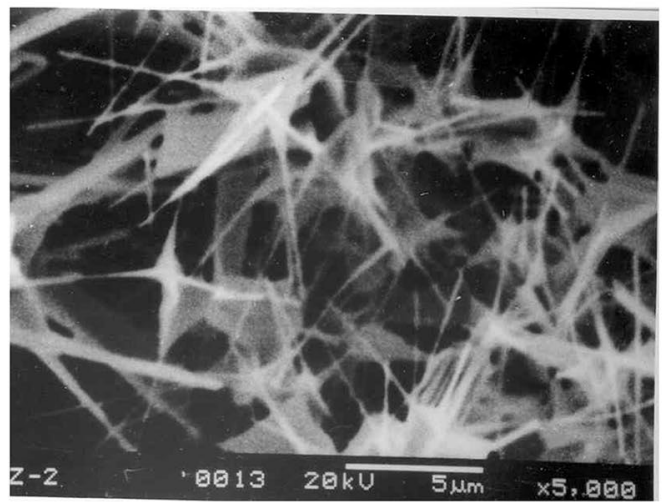

(c) 
the samples is similar. All the samples are composed of an interconnected network of tetrapods. Near the junction of four legs thin two dimensional structures are attached, which may be called as nanosheets. These nanosheets may further enhance the overall surface to volume ratio which is considered so crucial in several applications and have been proved superior in gas sensors [28], photoelectrochemical water splitting [20] etc. The general structure of these three samples is almost same namely tetrapod structure with growth of nanosheets on the individual legs. The legs of adjacent tetrapods join each other to form a highly porous interconnected structure.

\subsection{XRD characteristics}

The XRD pattern of the samples Z1 and Z3 is displayed in Fig. 3. All the visible peaks in the XRD patterns have been matched with ZnO JCPDS card number 790207 and suitably indexed confirming the wurtzite $\mathrm{ZnO}$ structure of the grown samples. The absence of diffraction peaks related to any other impurity confirms the high purity of the synthesized materials. Diffraction peaks are sharp with narrow full width at half maxima for Z3, while broader and less intense peaks for sample Z1 suggest smaller nanostructures. Peak indexing is shown in Table 1. The shifting of peaks towards lower Bragg angle, as noticed in sample $Z 1$, is generally attributed to small size induced lattice strain.

It is worthy to notice the comparatively strong line corresponding to $\mathrm{ZnO}$ (101) which indicates that (101) direction is the preferred orientation for the growth of both the samples $\mathrm{Z} 1$ and $\mathrm{Z3}$.

For hexagonal system the relation between $\mathrm{d}$ spacing and lattice parameters is given below $[29,30]$

$\frac{1}{d_{h k l}^{2}}=\frac{4}{3 a^{2}}\left(h^{2}+h k+k^{2}\right)+\frac{l^{2}}{c^{2}}$

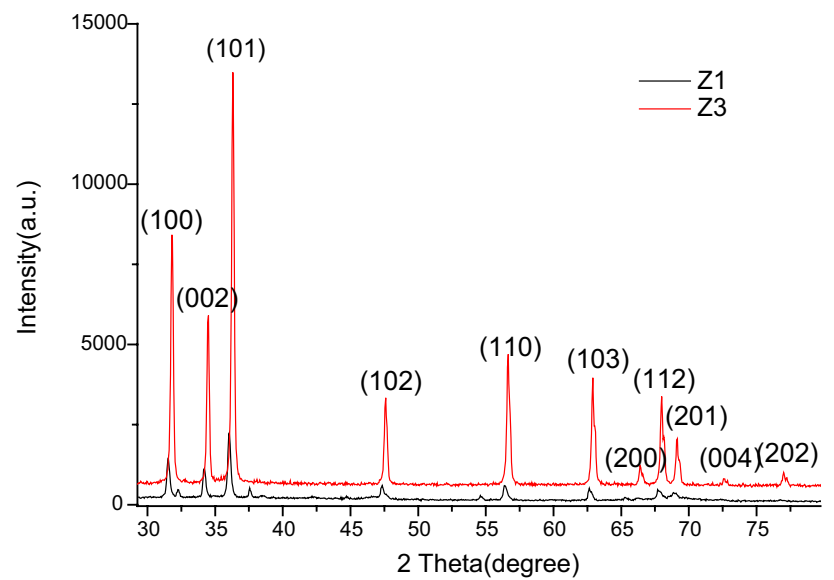

Fig. 3 XRD spectrum of zinc oxide nanostructures
Table 1 XRD analysis of $\mathrm{ZnO}$ nanostructures

\begin{tabular}{llllll}
\hline Sr. no. & Index (hkl) & (hkil) & Z3 & Z1 & 790207 \\
\hline 1 & $(100)$ & $(10 \overline{1} 0)$ & 31.80 & 31.55 & 31.699 \\
2 & $(002)$ & $(0002)$ & 34.50 & 34.20 & 34.382 \\
3 & $(101)$ & $(10 \overline{1} 1)$ & 36.30 & 36.05 & 36.182 \\
4 & $(102)$ & $(10 \overline{1} 2)$ & 47.60 & 47.35 & 47.459 \\
5 & $(110)$ & $(11 \overline{2} 0)$ & 56.65 & 56.40 & 56.463 \\
6 & $(103)$ & $(10 \overline{1} 3)$ & 62.90 & 62.65 & 62.760 \\
7 & $(200)$ & $(20 \overline{2} 0)$ & 66.40 & 66.25 & 66.216 \\
8 & $(112)$ & $(11 \overline{2} 2)$ & 68.00 & 67.70 & 67.805 \\
9 & $(201)$ & $(20 \overline{2} 1)$ & 69.15 & 68.85 & 68.924 \\
10 & $(004)$ & $(0004)$ & 72.60 & 72.35 & 72.472 \\
11 & $(202)$ & $(10 \overline{1} 0)$ & 77.00 & 76.75 & 76.786 \\
\hline
\end{tabular}

For first order diffraction

$2 d \sin \theta=\lambda$

Using Eqs. (1) and (2) for (100) plane

$a=\frac{\lambda}{\sqrt{3} \sin \theta}$

For (002) plane

$c=\frac{\lambda}{\operatorname{Sin} \theta}$

The unit cell parameters are presented in Table 2 for samples $Z 1$ and Z3. The average crystallite size was estimated using Debye-Scherrer equation

$D=\frac{K \lambda}{\beta \operatorname{Cos} \theta}$

where $\beta$ is full width at half maxima, $\lambda$ is the wavelength of $X$-ray $(=0.154 \mathrm{~nm}), \mathrm{K}$ is a constant which equals to 0.94 and $\theta$ is peak position. The average crystallite size $D$ for $Z 1$ and $Z 3$ sample was calculated as 31.5 and $35.5 \mathrm{~nm}$ respectively. The crystallite size is considered to be size of a coherently diffracting volume and does not particularly illustrate the exact morphological information and TEM analysis would present clearer picture.

\subsection{EDAX analysis}

The elemental analysis of nanotetrapods was done by EDAX study. Figure 4 depicts the elemental composition

Table 2 Calculated unit cell parameters of prepared materials

\begin{tabular}{llllll}
\hline Sample & $\theta$ for (100) & $a=\frac{\lambda}{\sqrt{3} \sin \theta}$ & $\theta$ for $(002)$ & $c=\frac{\lambda}{\sin \theta}$ & c/a ratio \\
\hline Z1 & 15.775 & 3.2705 & 17.1 & 5.2374 & 1.6014 \\
Z3 & 15.9 & 3.2454 & 17.24 & 5.1961 & 1.6011 \\
\hline
\end{tabular}


Fig. 4 Elemental analysis of sample Z3

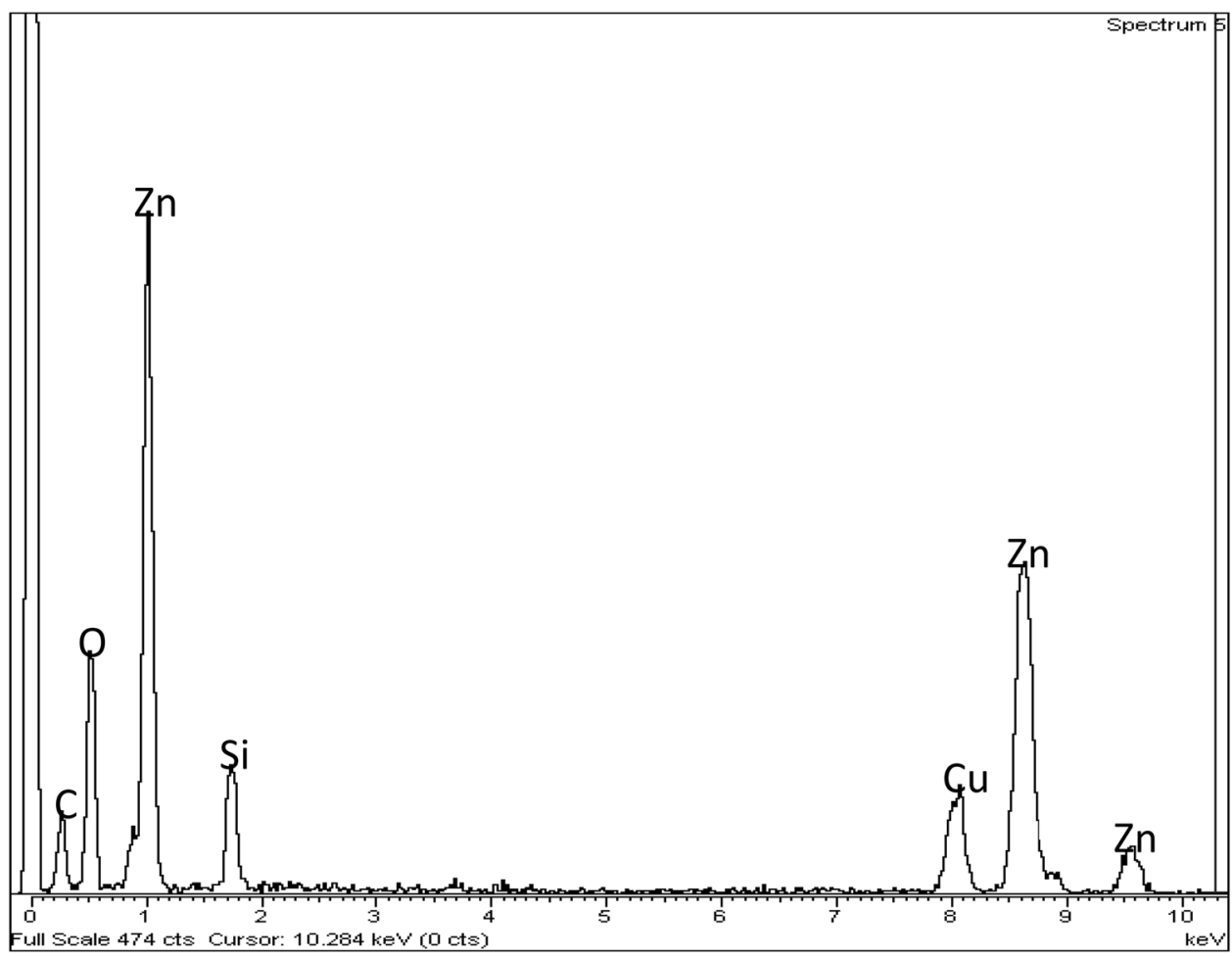

of the grown nanostructures for sample Z3. A weak Si peak observed in the spectrum can be safely attributed to the silicon substrate. $\mathrm{Cu}$ and $\mathrm{C}$ peaks are also detected and can be attributed to carbon coated copper grid used for TEM analysis. So the sample can be concluded as $\mathrm{ZnO}$.

\subsection{TEM study}

In order to better study the structure of as grown samples TEM and HRTEM analysis was done for sample Z1 and Z3. Figure 5 depicts the transmission electron micrographs of sample Z3. Figure 5 a clearly shows an individual tetrapod. It consists of 4 nanorods of high aspect ratio, with thickness about $35 \mathrm{~nm}$ at the joint to less than $10 \mathrm{~nm}$ towards the end. Some broken legs of other tetrapods and shredded nanosheet pieces are also visible in the image. An individual leg of the tetrapod is very clear beneath the nanosheet. It can be inferred from this transparency that nanosheets are very fine two dimensional nanostructures presenting very high surface to volume ratio, again indicating that it can be highly suitable material for gas sensing applications and other applications where high surface area is crucial. Figure $5 \mathrm{~b}$ presents the broader view of the
Fig. 5 a An individual tetrapod. $\mathbf{b}$ Broader view of sample Z3

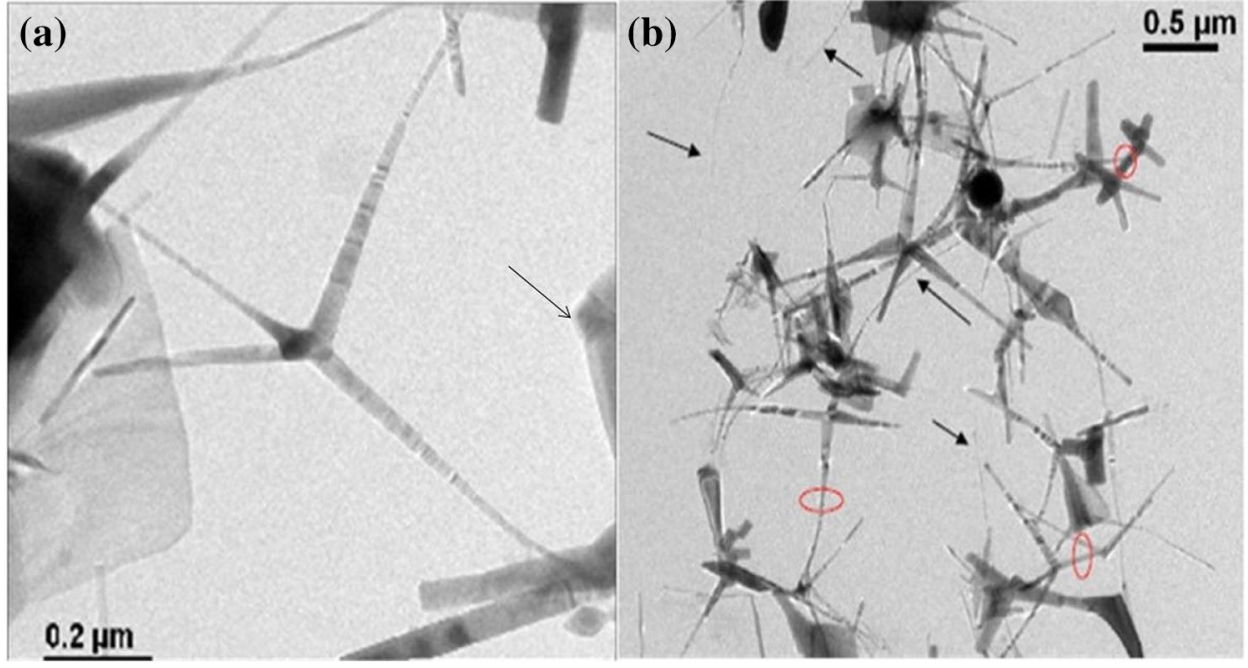

SN Applied Sciences A SPRINGER NATURE journa 
sample. It can be implied from Fig. $5 \mathrm{~b}$ that the length of an individual leg is $1 \mu \mathrm{m}$ to $1.5 \mu \mathrm{m}$. Although, ultrasonication process during sample preparation may have altered the morphology to some extent, nevertheless, two prominent features are conspicuous. The first being the general narrowing down of the legs towards the end indicated by arrows, which is generally termed as terminal bottleneck in literature. The second is the joining of two legs of adjacent tetrapods pointed out by circles, being referred as terminal coalescence in literature. Both these features are considered as the identifying characteristics of vapour solid growth mechanism [31]. The nanosheets were found grown at the joints of tetrapod legs and there is a general narrowing of legs towards the end which is the hallmark of the VS growth [31].

Zinc has boiling point about $910^{\circ} \mathrm{C}$. At higher temperatures, zinc evaporates and nucleation starts owing to collision between various species available. The zinc vapours formed by burning of zinc pellets are carried away towards cooler areas and react with oxygen and start nucleation. Tetrapods then grow from this core nucleus. This process does not require any catalyst and purer sample are obtained.

The hint of a twin boundary can be observed at the centre of a tetraleg indicated by an arrow in Fig. 5a. Octatwin nucleus model has been proposed for similar products [32]. In this study, it is proposed that as different size and densities of structures are formed, these structures are naturally transported to different positions according to the corresponding densities. The finest of structures (Z1) are lightest and dispersed by fumes in the chamber and later get settled. The next denser structures (Z3) are carried to the top towards the $\mathrm{Si}$ substrate. The heaviest products (Z2) remained near tungsten basket. So the products are automatically segregated according to the corresponding density. It is suggested that the flow of an inert gas can be utilised to control and manipulate this natural segregation and collection of various sized products. The growth was carried out inside bell jar of a vacuum coating unit with limited oxygen supply. As various types of products were obtained, the yield of products may depend upon the variation of available parameters. One obvious choice may be oxygen flow rate which can be further manipulated and optimized.

Figure 6 represents an individual tetrapod of sample Z1. The thickness of a single nanorod at the junction is nearly $20 \mathrm{~nm}$ and towards the end it is about $9 \mathrm{~nm}$. The length of a leg is about $80 \mathrm{~nm}$ as visible in the Fig. 6 . Figure 7a represents HRTEM image of an individual leg of a tetrapod. The single crystalline atomic planes with $\mathrm{d}$ spacing of $0.26 \mathrm{~nm}$ are clearly visible. This $d$ spacing corresponds to (0002) ZnO planes consequently, the growth direction of this particular leg is (0001) [33]. Figure $7 b$ shows the

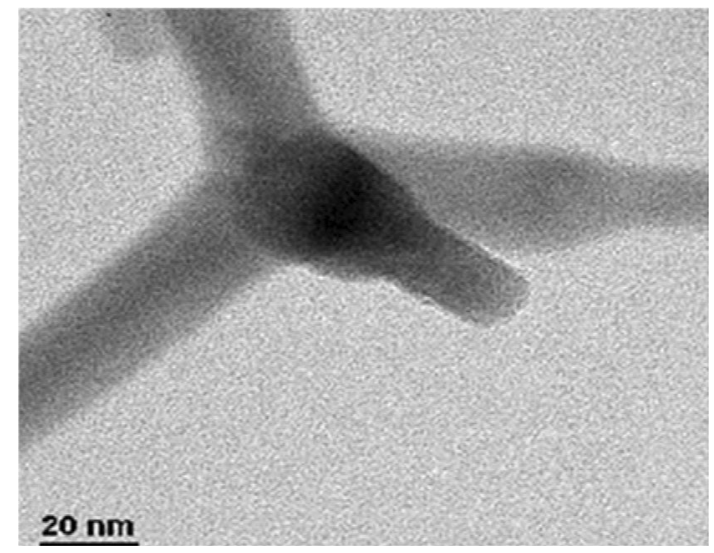

Fig. 6 TEM image of an individual tetrapod in sample Z1
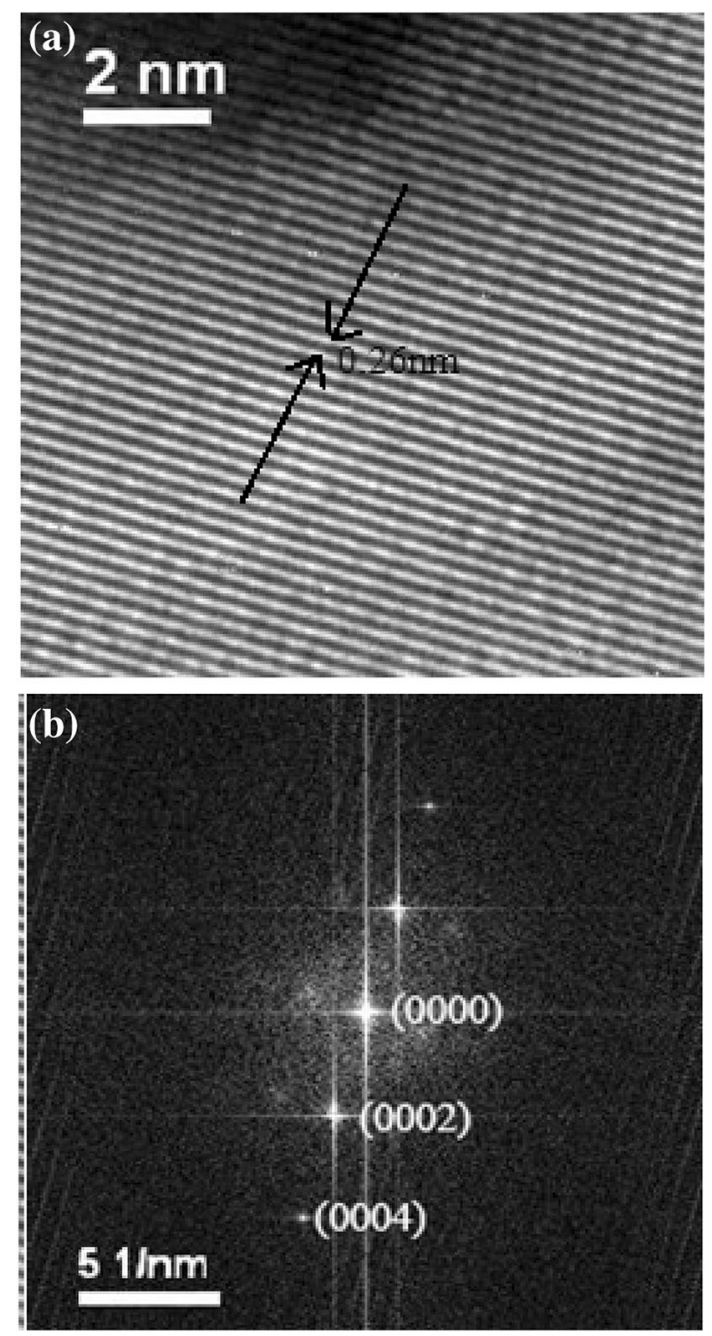

Fig. 7 a HRTEM of a single leg of tetrapod. b The corresponding FFT of HRTEM of ZnO material 
corresponding Fast Fourier Transform (FFT) of HRTEM. The FFT shows that the selected area is single crystalline. The spots are indexed according to the $d$ spacing as measured from the FFT.

\subsection{Photoluminescence characteristics}

Figure 8 presents the room temperature photoluminescence emission spectra (with excitation wavelength $320 \mathrm{~nm}$ ) of sample Z1, Z2, Z3 and PL emission of bulk zinc oxide powder (Merck $99 \%$ pure) is shown for comparison. All the samples confirm typical $\mathrm{ZnO}$ peaks. The UV band around $385 \mathrm{~nm}$ corresponds to near band edge emission, a violet band observed near $420 \mathrm{~nm}$ is attributed to the recombination of holes of valence band and electrons present at zinc interstitials. The transition between zinc interstitials and zinc vacancies is responsible for blue band around $480 \mathrm{~nm}$ [34]. Generally, it is assumed that high UV to visible emission corresponds to high quality of the sample and a low UV to visible ratio represents lattice defects. The UV to visible emission ratio is very high for Z1, higher than the standard bulk sample for $Z 3$ and comparable to bulk sample for $Z 2$. The smaller dimensions of nanostructures make the occurrence of zinc interstitials and vacancies less probable statistically. This extra purity owing to statistical probability can be observed in this study. Sample Z1, which contain smaller nanostructures, show very high intensity of band edge UV emission in comparison with defect originated visible band. Therefore, the high optical quality of the sample $Z 1$ can be explained and the quality deteriorates as the size of nanostructures increase in Z3 and further in Z2.

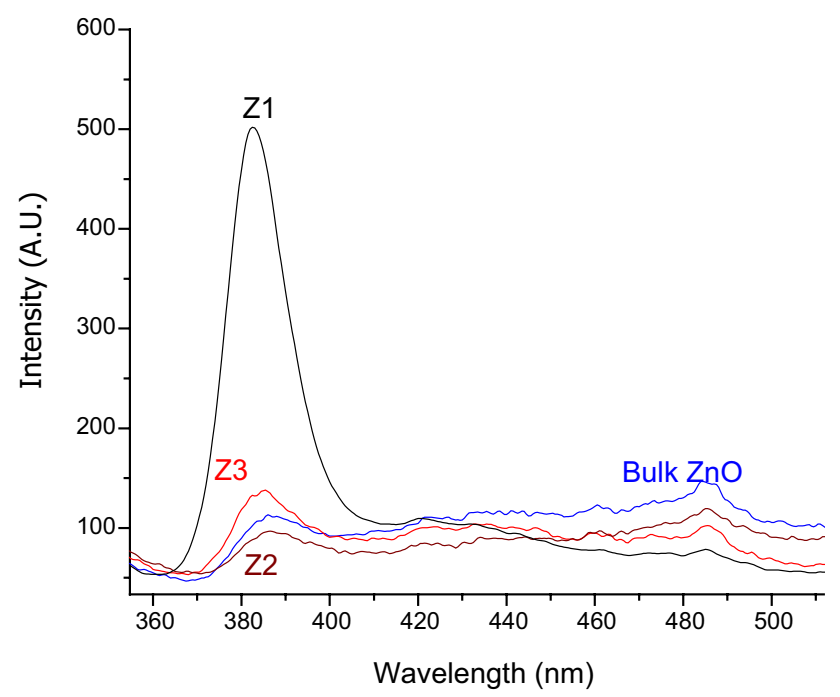

Fig. 8 Comparison PL of samples Z1, Z2 and Z3 with bulk $\mathrm{ZnO}$ (standard powder)
The blue shifting of near band edge UV emission is clearly visible when $\mathrm{PL}$ spectrum of $\mathrm{Z} 1$ is compared with that of standard $\mathrm{ZnO}$ sample. The quantum confinement theory explains this shift as it predicts increase in band gap with decrease in crystal size [35].

The formula for band gap considering quantum confinement is given by

$E_{g}=E_{g 0}+\frac{h^{2} \pi^{2} \mu}{2 d^{2}}-\frac{1.8 e^{2}}{\varepsilon d}$

where $E_{g}$ is band gap for bulk $\mathrm{ZnO}$, $d$ is particle size and $\mu$ is reduced mass.

$\frac{1}{\mu}=\frac{1}{m_{e}}+\frac{1}{m_{h}}$

where $\mathrm{m}_{\mathrm{e}}=0.38 \mathrm{~m}_{0}$ and $\mathrm{m}_{\mathrm{h}}=1.80 \mathrm{~m}_{0}$ are the electron and hole effective masses, respectively, $\varepsilon=8.75$, is the dielectric constant [36].

The Eq. (6) is reduced to

$E_{g}=E_{g 0}+\frac{75.885}{d^{2}(\mathrm{~nm})}-\frac{1.902}{d(\mathrm{~nm})}$

The peak shifting of $382.5 \mathrm{~nm}$ from $385.5 \mathrm{~nm}$ corresponds to $0.025 \mathrm{eV}$ shift in band gap, which implies a crystal size of $29 \mathrm{~nm}$ for sample Z1, which is in good agreement with crystal size $31.5 \mathrm{~nm}$ obtained by Scherer formula. This shifting effect in Eq. (8) reduces rapidly with increase in crystal size. For sample Z3, shifting of only $1 \mathrm{~nm}$ is observed from 385.5 to $384.5 \mathrm{~nm}$. This corresponds to a shifting of $0.008 \mathrm{eV}$, in the band gap and it in turn indicates a crystallite size of $34 \mathrm{~nm}$ which is in excellent agreement with crystallite size of $35 \mathrm{~nm}$ observed by Scherer formula.

\section{Conclusion}

In summary, the nanosheets attached nanotetrapods grown were of three different sizes, sample Z2 were microstructures of largest dimension, Z3 nanotetrapods of smaller size and Z1 smallest nanotetrapods. The size of nanotetrapods was decreased as distance from tungsten basket was increased presenting a way to control the size as per the desired characteristics. XRD analysis exhibited pure wurtzite structure and average crystallite size of 31.5 and $35.5 \mathrm{~nm}$ for sample $\mathrm{Z1}$ and $\mathrm{Z} 3$ respectively. EDAX analysis also confirmed the purity of the prepared sample. TEM analysis recommended that the growth mechanism is vapour-solid growth and presented a clear view as well as dimensions of individual nanotetrapods. Photoluminescence study also confirmed the purity of the sample, as the near band edge emission was several times higher than the defect induced visible emission. Also, the 
photoluminescence peak shifting confirmed the average crystallite size of particles $29 \mathrm{~nm}$ for sample Z1 and $34 \mathrm{~nm}$ for sample Z3, which are in excellent agreement with 31.5 and $35 \mathrm{~nm}$ respectively as size estimated by the XRD analysis. The synthesised materials of nanotetrapods having promising properties could be applied in various thin films for the desired applications.

\section{Compliance with ethical standards}

Conflict of interest The authors declare that they have no conflict of interest.

\section{References}

1. Bai $P$, Zhu G, Lin ZH, Jing Q, Chen J, Zhang G, Ma J, Wang ZL (2013) Integrated multilayered triboelectric nanogenerator for harvesting biomechanical energy from human motions. ACS Nano 7:3713

2. Chaudhary S, Umar A, Bhasin KK, Baskoutas S (2018) Chemical sensing applications of $\mathrm{ZnO}$ nanomaterials. Materials 11:287

3. Wang ZL (2008) Splendid one-dimensional nanostructures of zinc oxide: a new nanomaterial family for nanotechnology. ACS Nano 2:1987

4. Alsultany FH, Hassan Z, Ahmed NM (2016) Large-scale uniform ZnO tetrapods on catalyst free glass substrate by thermal evaporation method. Mater Res Bull 79:63

5. Laurenti M, Stassi S, Canavese G, Cauda V (2017) Surface engineering of nanostructured $\mathrm{ZnO}$ surfaces. Adv Mater Interfaces 4:1600758

6. Ta HQ, Zhao L, Pohl D, Pang J, Trzebicka B, Rellinghaus B, Pribat D, Gemming T, Liu Z, Bachmatiuk A, Rümmeli MH (2016) Graphene-like ZnO: a mini review. Crystals 6:100

7. Wang M, Li AD, Kong JZ, Gong YP, Zhao C, Tang YF, Wu D (2018) Fabrication and characterization of $\mathrm{ZnO}$ nano-clips by the polyol-mediated process. Nanoscale Res Lett 13:47

8. Wang $X$, Ahmad M, Sun H (2017) Three-dimensional ZnO hierarchical nanostructures: solution phase synthesis and applications. Materials 10:1304

9. Zhang Y, Wu Y, Ding H, Yan Y, Zhou Z, Ding Y, Liu N (2018) Sealing $\mathrm{ZnO}$ nanorods for deeply rechargeable high-energy aqueous battery anodes. Nano Energy 53:666

10. Waghadkar Y, Shinde M, Rane S, Gosavi S, Terashima C, Fujishima A (2018) Highly efficient dye-sensitized solar cells by $\mathrm{TiCl}_{4}$ surface modification of $\mathrm{ZnO}$ nano-flower thin film. J Solid State Electrochem 22:3621

11. Luo J, Wang $Y$, Zhang Q (2018) Progress in perovskite solar cells based on ZnO nanostructures. Sol Energy 163:289

12. Trino LD, Dias LFG, Albano LGS, Bronze-Uhlea ES, Rangel EC, Graeff CFO, Lisboa-Filho PN (2018) Zinc oxide surface functionalization and related effects on corrosion resistance of titanium implants. Ceram Int 44:4000

13. Sirelkhatim A, Mahmud S, Seeni A, Kaus NHM, Ann LC, Bakhori SKM, Hasan H, Mohamad D (2015) Review on zinc oxide nanoparticles: antibacterial activity and toxicity mechanism. Nano-Micro Lett 7:219

14. Gupta J, Bahadur D (2018) Defect-mediated reactive oxygen species generation in Mg-substituted $\mathrm{ZnO}$ nanoparticles: efficient nanomaterials for bacterial inhibition and cancer therapy. ACS Omega 3:2956

15. Zhang W, Zhang L, Gao H, Yang W, Wang S, Xing L, Xue X (2018) Self-Powered implantable skin-like glucometer for real-time detection of blood glucose level in vivo. Nano-Micro Lett 10:32

16. $\mathrm{Xu} \mathrm{CL}$, Wang $\mathrm{YZ}$ (2017) One-step approach to the growth of $\mathrm{ZnO}$ nano-/microrods on cellulose toward its durable superhydrophobicity. Adv Mater Interfaces 4:1700550

17. Ahmad R, Tripathy N, Ahn M, Hahn Y (2017) Solution process synthesis of high aspect ratio $\mathrm{ZnO}$ nanorods on electrode surface for sensitive electrochemical detection of uric acid. Sci Rep 7:46475

18. Boon C, Yong L, Wahab A (2018) A review of $\mathrm{ZnO}$ nanoparticles as solar photocatalysts: synthesis, mechanisms and applications. Renew Sustain Energy Rev 81:536

19. Kong L, Tu K, Guan H, Wang X (2017) Growth of high-density $\mathrm{ZnO}$ nanorods on wood with enhanced photostability, flame retardancy and water repellency. Appl Surf Sci 407:479

20. Hou T, Boppella R, Shanmugasundaram A (2017) Hierarchically self-assembled $\mathrm{ZnO}$ architectures: establishing light trapping networks for effective photoelectrochemical water splitting. Int J Hydrogen Energy 42:15126

21. Mishra YK, Adelung R (2018) ZnO tetrapod materials for functional applications. Mater Today 21:631

22. Yan L, Uddin A, Wang $\mathrm{H}$ (2015) ZnO tetrapods: synthesis and applications in solar cells. Nanomater Nanotechnol 5:19

23. Antoine TE, Hadigal SR, Yakoub AM, Mishra YK, Bhattacharya P, Haddad C, Valyi-Nagy T, Adelung R, Prabhakar BS, Shukla D (2016) Intravaginal zinc oxide tetrapod nanoparticles as novel immunoprotective agents against genital herpes. J Immunol 196:4566

24. Calestani D, Mosca R, Zanichelli M, Villani M, Zappettini A (2011) Aldehyde detection by ZnO tetrapod-based gas sensors. J Mater Chem 21:15532

25. Gedamu D, Paulowicz I, Kaps S, Lupan O, Wille S, Haidarschin G, Mishra YK, Adelung R (2014) Rapid fabrication technique for interpenetrated $\mathrm{ZnO}$ nanotetrapod networks for fast UV sensors. Adv Mater 26:1541

26. Wu C, Kim TW, Guo T, Li F (2016) Unique visible-light-assisted field emission of tetrapod-shaped $\mathrm{ZnO}$ /reduced grapheneoxide core/coating nanocomposites. Sci Rep 6:38613

27. Jin X, Deng M, Kaps S, Zhu X, Holken I, Mess K, Adelung R, Mishra YK (2014) Study of tetrapodal ZnO-PDMS composites: a comparison of fillers shapes in stiffness and hydrophobicity improvements. PLoS ONE 9:e106991

28. Kaneti YV, Yue J, Chuyang C (2014) Crystal plane-dependent gassensing properties of zinc oxide nanostructures: experimental and theoretical studies. Phys Chem Chem Phys 16:11471

29. Bindu $P$, Thomas $S$ (2014) Estimation of lattice strain in ZnO nanoparticles: X-ray peak profile analysis. J Theor Appl Phys 8:123

30. Zak AK, Majid WHA, Abrishami ME, Yousefi R (2011) X-ray analysis of $\mathrm{ZnO}$ nanoparticles by Williamson-Hall and size-strain plot methods. Solid State Sci 13:251

31. Zhang Y, Jia H, Luo X, Chen X, Yu D, Wang R (2003) Synthesis, microstructure, and growth mechanism of dendrite $\mathrm{ZnO}$ nanowires. J Phys Chem B 107:8289

32. Dai Y, Zhang Y, Wang ZL (2003) The octa-twin tetraleg ZnO nanostructures. Solid State Commun 126:629

33. Tsukazaki A, Ohtomo A, Onuma T, Ohtani M, Makino T, Sumiya M, Ohtani K, Chichibu SF, Fuke S, Segawa Y, Ohno H, Koinuma H, Kawasak M (2005) Repeated temperature modulation epitaxy for p-type doping and light-emitting diode based on $\mathrm{ZnO}$. Nat Mater 4:42 
34. Peter IJ, Praveen E, Vignesh G, Nithiananthi P (2017) ZnO nanostructures with different morphology for enhanced photocatalytic activity. Mater Res Express 4:124003

35. Cho S, Ma J, Kim Y, Sun Y, Wong GKL, Ketterson JB (1999) Photoluminescence and ultraviolet lasing of polycrystalline $\mathrm{ZnO}$ thin films prepared by the oxidation of the metallic Zn. Appl Phys Lett 75:2761
36. Dietz RE, Hopfield JJ, Thomas DG (1961) Excitons and the absorption edge of ZnO. J Appl Phys 32:2282

Publisher's Note Springer Nature remains neutral with regard to jurisdictional claims in published maps and institutional affiliations. 